\title{
Efficacy, safety, and cost-effectiveness analysis of adjuvant herbal medicine treatment, Palmijihwang-hwan, for chronic low back pain: a study protocol for randomized, controlled, assessor-blinded, multicenter clinical trial
}

Won-Suk Sung ${ }^{1}$, Sae-Rom Jeon ${ }^{2}$, Ye-Jin Hong², Tae-Hun Kim³ ${ }^{3}$ Seungwon Shin ${ }^{3}$, Hyun-Jong Lee ${ }^{4}$, Byung-Kwan Seo ${ }^{5}$, Yeon-Cheol Park', Eun-Jung Kim ${ }^{1 *}$ and Dong-Woo Nam ${ }^{5 *}$

\begin{abstract}
Background: Low back pain is a common symptom and continuous or recurrent pain results in chronic low back pain (CLBP). While many patients with CLBP have tried various treatments, complementary and alternative medicine including acupuncture and herbal medicine is one of the commonly used treatments. Palmijihwang-hwan is a herbal medicine used frequently in clinical practice but there has been no report of the efficacy, safety, or costeffectiveness analysis of Palmijihwang-hwan for CLBP.

Methods: This study is a randomized, assessor-blinded, multicenter, clinical trial with two parallel groups. Four Korean medicine hospitals will recruit 84 participants and randomly allocate them into the control or treatment group in a 1:1 ratio. The control group will receive acupuncture treatment at 11 local and 4 distal acupuncture points for 20 min twice a week for 6 weeks. The treatment group will receive the same acupuncture treatment as the control group and also take Palmijihwang-hwan for 6 weeks. The primary outcome will be the change in visual analog scale (VAS) score between baseline (visit 1) and completion of the intervention (visit 12), and secondary outcomes will be pain-related clinical relevance (minimal clinical important difference or the proportion of the participants who decrease more than 30, or 50\% on VAS), disability (Roland and Morris Disability Questionnaire), quality of life (EuroQol-5D), global assessment (Patient Global Impression of Change), and economic analysis (costeffectiveness and cost-utility analysis). Additionally, safety will be assessed.
\end{abstract}

Discussion: The results of our study will provide the clinical evidence about the efficacy, safety, and costeffectiveness analysis of Palmijihwang-hwan for CLBP. There will be a chance to provide multiple subdivided influence of this treatment with various outcome measures, but lack of placebo is our limitation.

(Continued on next page)

\footnotetext{
*Correspondence: hanijjung@naver.com; hanisanam@daum.net

'Department of Acupuncture \& Moxibustion, Dongguk University Bundang Oriental Hospital, Bundang-gu, Seongnam-si, Gyeonggi-do 13601, South Korea

${ }^{5}$ Department of Acupuncture \& Moxibustion, College of Korean Medicine, Kyung Hee University, 26 Kyungheedae-ro, Dongdaemun-gu, Seoul 02447, South Korea

Full list of author information is available at the end of the article
}

(c) The Author(s). 2019 Open Access This article is distributed under the terms of the Creative Commons Attribution 4.0 International License (http://creativecommons.org/licenses/by/4.0/), which permits unrestricted use, distribution, and reproduction in any medium, provided you give appropriate credit to the original author(s) and the source, provide a link to the Creative Commons license, and indicate if changes were made. The Creative Commons Public Domain Dedication waiver (http://creativecommons.org/publicdomain/zero/1.0/) applies to the data made available in this article, unless otherwise stated. 
(Continued from previous page)

Trial registration: Clinical Research Information Service, KCT0002998. Registered on 12 July 2018.

Keywords: Chronic low back pain, Palmijihwang-hwan, Randomized controlled trial, Study protocol

\section{Background}

Low back pain (LBP) is a symptom that $84 \%$ of humans experience at some time in their life [1]. LBP can be classified according to its duration into acute $(<6$ weeks), subacute (6-12 weeks), and chronic ( $>12$ weeks) [2]. Most patients with acute LBP recover within 4 weeks but recurrences are common [3]. In the case of insufficient treatment and management, continuous pain results in chronic LBP (CLBP), which is known to be related to various factors including psychological distress and psychiatric disorders [4]. Deficits in and poor satisfaction with conventional treatments [5] for CLBP have led to attempts to treat CLBP using nonpharmacologic [6] or complementary and alternative medicine (CAM).

In Korean medicine, several treatments including acupuncture [7], electroacupuncture [8], and pharmacopuncture such as bee venom [9] have been examined as a treatment for LBP, and several studies have reported therapeutic effects. Particularly, acupuncture is a wellknown treatment for CLBP. Various studies have reported on the effects on pain [10] and the pragmatic aspects [11] of acupuncture in treating CLBP and some have suggested that acupuncture has more effect on the reduction of pain and bothersomeness than sham control in patients with CLBP [12]. Several studies have investigated the clinical effect of acupuncture combined with other treatments such as direct moxibustion [13], physiotherapy [14], and pharmacopuncture [15]. However, studies on the effects of adjuvant Korean herbal medicines on CLBP, particularly in combination with acupuncture, are rare.

Among them, Palmijihwang-hwan (Ba-Wei-DiHuang-Wan in Chinese and Hachimi-jio-gan in Japanese) has been reported to be useful for skeletal muscle proliferation [16] and to be helpful for patients with sciatic neuralgia or lumbago with bad circulation in the lower body [17], which is similar to the symptoms of CLBP. Eight herbs used in Palmijihwang-hwan are also reported to have analgesic effects in experimental studies. It is reported that Aconitum carmichaelii improves inflammatory conditions by mediating TNFalpha response [18] and processed A. carmichaelii exerts an analgesic effect via central opioid receptors [19]. Catapol, an ingredient in Rehmannia glutinosa, alleviates neuropathic pain with the modulation of neuroinflammation in the spinal cord in rats [20]. Cornus officinalis is reported to have anti-inflammatory and analgesic effects [21], while Dioscorea batatas is confirmed to have anti-inflammatory activity through the inhibition of iNOS and COX-2 expression [22]. Scientific studies indicate the anti-inflammatory effects of Alisma orientale [23], Poria coccos [24], Paeonia sulffruticosa [25], and Cinnamomum cassia [26].

However, these reports include no clinical study to confirm the efficacy or safety of Palmijihwang-hwan or to include a cost-effectiveness analysis (CEA) of Palmijihwang-hwan, for treatment of CLBP. Therefore, we describe a protocol for a randomized controlled clinical trial that uses Palmijihwanghwan combined with acupuncture for treatment of CLBP.

\section{Methods and design Objectives}

The purpose of this study is to investigate the efficacy, safety, and CEA of Palmijihwang-hwan combined with acupuncture for treatment of CLBP by comparing this with the effects of acupuncture treatment alone.

\section{Design}

This study is a randomized, assessor-blinded, multicenter, clinical trial with two parallel groups. We intend to investigate the synergic effects of Palmijihwang-hwan with acupuncture treatment in patients with CLBP. Eligible participants will be assigned to one of two groups, the control group (only acupuncture) or the treatment group (Palmijihwang-hwan + acupuncture). The flowchart is as shown in Fig. 1. The Standard Protocol Items: Recommendations for Interventional Trials (SPIRIT) checklist is provided in Additional file 1.

\section{Sample size calculation}

We performed sample size calculations to determine the appropriate number of participants. The purpose of this study is to verify the synergic effect of the combination treatment (acupuncture + Palmijihwang-hwan) compared to acupuncture treatment alone, using a visual analog scale (VAS) score for change between baseline and completion of the intervention (6 weeks). However, no study has been conducted to investigate the effects of Palmijihwang-hwan combined with acupuncture in the treatment of CLBP. Thus, we reviewed a similar study conducted in patients with CLBP [27] in which the authors evaluated the efficacy of a herbal formula, Ojeoksan, by measuring the VAS score change between baseline and completion of the intervention (4 weeks) and comparing this with the placebo group in randomized clinical trials of CLBP. In that study, the change in VAS score between baseline and completion of the intervention was 


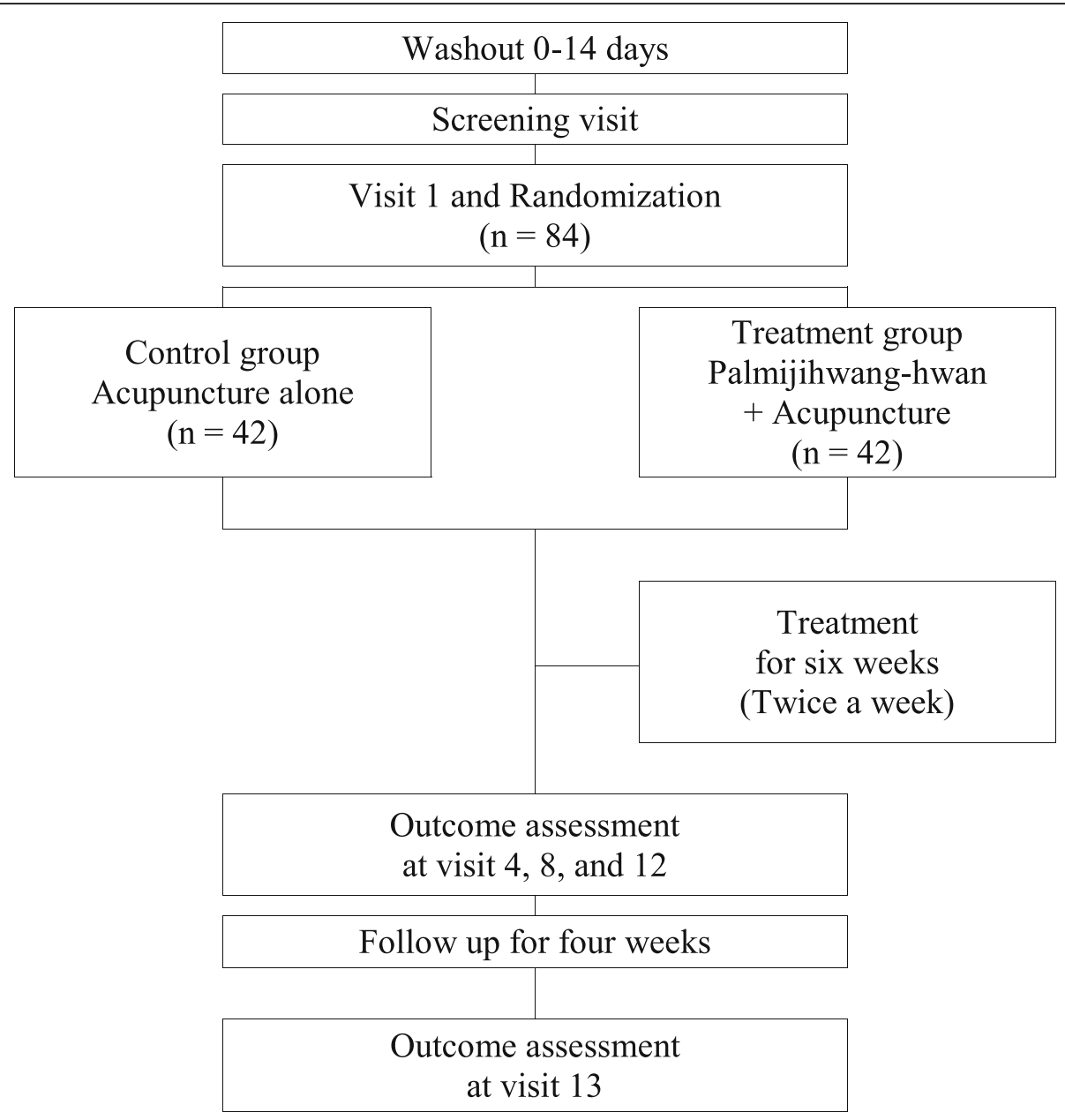

Fig. 1 Study flowchart

$-26.41 \pm 19.06$ in the treatment group and $-12.83 \pm 19.45$ in the control group. Based on these data, 33 participants would be required per intervention group for a two-arm study and a two-tailed test with 0.80 power $(1-\beta)$ at the $0.05 \alpha$ level, as calculated using $G *$ Power (Version 3.1.9.3) [28]. After assuming a 20\% drop out rate, we calculated that we would need to recruit 42 participants per group. Thus, a total of 84 participants will be required.

\section{Participants}

We will recruit 84 participants with CLBP and assign them randomly to the control group or the treatment group in a $1: 1$ ratio.

\section{Inclusion criteria}

\section{The inclusion criteria are:}

1. Patients with CLBP, aged $19-65$ years

2. Chief complaint of LBP lasting $>3$ months
3. Score $>4 \mathrm{~cm}$ on a $10-\mathrm{cm}$ VAS for LBP at one week prior to enrollment

4. Voluntarily agreeing to participation and observance of trials with written consent

\section{Exclusion criteria}

\section{The exclusion criteria are:}

1. Hypersensitive reactions to or history of reactions to Palmijihwang-hwan or its components

2. Abnormalities in the lower extremity on neurological examination

3. Requirement for surgical treatment because of severe neurological deficits (sensory or motor) or cauda equina syndrome

4. Previous spinal surgery or scheduled spinal surgery during the trial

5. Patients with scoliosis or neurodegenerative disease

6. Vertebral fracture, inflammatory spondylitis, spinal infection, or malignant tumor 
7. Pregnancy, lactation, plans to conceive, refusal to use appropriate contraception methods during the trial

8. Gastrointestinal disorders or diarrhea

9. Genetic disorders such as galactose intolerance, Lapp lactase deficiency, or glucose-galactose malabsorption

10. Use of acupuncture, herbal medicine, injection, physical therapy, or manipulation treatment for low back pain within two weeks

11. Chief complaint of part other than the lumbar region

12. Participants in other clinical studies related to CLBP within a month

13. Ineligibility for the trial as judged by the investigator

\section{Recruitment}

Four Korean medicine hospitals, Kyung Hee University Korean Medicine Hospital, Kyung Hee University Korean Medicine Hospital at Gangdong, Oriental Medicine Hospital of Daegu Haany University, and Dongguk University Bundang Oriental Hospital, will recruit participants through advertisements on bulletin boards, local newspapers, and public boards.

\section{Procedure}

The study schedule is as shown in Fig. 2. At the screening visit, patients who are interested in participation and who visit one of the four Korean medicine hospitals will be informed about the study. They will be also informed about expected benefits and risks and that they are able to withdraw from the study at any visit. If they sign the informed consent form voluntarily, the investigator will perform several examinations including check of vital signs, physical examination, spine $\mathrm{x}$-ray, blood chemistry test, pregnancy test, and will collect demographic data, CLBP history, and concomitant treatment data, and apply the VAS, to determine the patient's eligibility. If patients are eligible for the study but are taking medication, the investigator will determine the day of screening according to the participant's medication to give a washout period. If the participant is taking anti-inflammatory medication or painkillers, the investigator will give a 2week washout period. During the 6-week treatment, the participants in both groups will visit the hospital twice a week (total 12 visits) and receive acupuncture treatment at every visit. Participants in the treatment group will additionally receive Palmijihwang-hwan (at visit 1,4 , and 8) during the treatment period. Then, participants in both groups will be asked to complete a survey every 2 weeks (at visit 4, 8, and 12). On visit 13, 4 weeks after visit 12, all participants will visit the hospital for the final time.

\section{Randomization and allocation concealment}

A randomization sequence will be generated by an independent statistician who does not participate in this study, using R software (Version 3.5.0; R Foundation for

\begin{tabular}{|c|c|c|c|c|c|c|c|c|c|c|c|c|c|c|}
\hline & \multicolumn{14}{|c|}{ Study period } \\
\hline & \multirow{2}{*}{$\begin{array}{c}\text { Enrollment } \\
\mathbf{- 2} \sim \mathbf{0} \\
\end{array}$} & \multicolumn{12}{|c|}{ Intervention } & \multirow{3}{*}{$\begin{array}{c}\text { Follow-up } \\
\mathbf{1 0} \\
13\end{array}$} \\
\hline Time point (week) & & \multicolumn{2}{|c|}{1} & \multicolumn{2}{|c|}{2} & \multicolumn{2}{|r|}{3} & \multicolumn{2}{|c|}{4} & \multicolumn{2}{|c|}{5} & \multicolumn{2}{|c|}{6} & \\
\hline Time point (visit) & Screening & 1 & 2 & 3 & 4 & 5 & 6 & 7 & 8 & 9 & 10 & 11 & 12 & \\
\hline \multicolumn{15}{|l|}{ Enrollment: } \\
\hline Eligibility screen & $\mathrm{X}$ & & & & & & & & & & & & & \\
\hline Informed consent & $\mathrm{X}$ & & & & & & & & & & & & & \\
\hline Allocation & & $\mathrm{X}$ & & & & & & & & & & & & \\
\hline \multicolumn{15}{|l|}{ Interventions: } \\
\hline \multicolumn{15}{|l|}{ Palmijihwang-hwan treatment } \\
\hline Acupuncture treatment & & 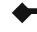 & & & & & & & & & & & $\rightarrow$ & \\
\hline \multicolumn{15}{|l|}{ Assessments: } \\
\hline VAS & $\mathrm{X}$ & $\mathrm{X}$ & & & $\mathrm{X}$ & & & & $\mathrm{X}$ & & & & $\mathrm{X}$ & $\mathrm{X}$ \\
\hline RMDQ & & $\mathrm{X}$ & & & $\mathrm{X}$ & & & & $\mathrm{X}$ & & & & $\mathrm{X}$ & $\mathrm{X}$ \\
\hline EQ-5D & & $\mathrm{X}$ & & & $\mathrm{X}$ & & & & $\mathrm{X}$ & & & & $\mathrm{X}$ & $\mathrm{X}$ \\
\hline PGIC & & & & & & & & & & & & & $\mathrm{X}$ & $\mathrm{X}$ \\
\hline \multicolumn{15}{|l|}{ Economic analysis } \\
\hline Adverse events & & 8 & & & & & & & & & & & & \\
\hline Credibility test & & $\mathrm{X}$ & & & & & & & & & & & & \\
\hline
\end{tabular}

Fig. 2 Study schedule (Standard Protocol Items: Recommendations for Interventional Trials, SPIRIT). Abbreviations: EQ-5D: EuroQol-5 dimension; PGIC: patient global impression of change; RMDQ: Roland and Morris disability questionnaire; VAS: visual analog scale 
Statistical Computing, Vienna, Austria). Randomization will be stratified based on four Korean medicine hospitals, and then block randomization will be used to approximately equalize the number of participants in each group. A random code will be delivered in the sealed envelopes, in which the allocated group is written. A delegated investigator in each site will open the envelope in sequence and allocate a participant to either intervention or control arm, only when all the screening criteria are satisfied. Allocation will be concealed until the investigator opens each sealed envelope. The randomization sequence will be kept by the independent statistician or physician during the clinical trial period and concealed until the occurrence of the event predefined as a reason for code-breaking.

\section{Blinding}

The additional Palmijihwang-hwan treatment means that the participants and practitioners cannot be blinded; thus, the practitioners who perform the acupuncture treatment or provide Palmijihwang-hwan will not assess the outcome. The assessor who record case report form (CRF) will be instructed to ask questions simply and write in detail the participant's answer. Both of the assessor and participant are prevented from talking about the treatment.

\section{Interventions}

\section{Control group intervention}

Participants in the control group will receive only the acupuncture treatment. Sterilized stainless steel needles with $0.25 \mathrm{~mm}$ width and $40 \mathrm{~mm}$ length (DB108C; Dongbang Medical Co., Boryung-si, South Korea) will be inserted at 11 local and 4 distal acupuncture points for $20 \mathrm{~min}$. Acupuncture points are as shown in Table 1 . Participants will receive the acupuncture treatment twice a week for 6 weeks, performed by Korean medical doctors who are specialists in acupuncture and moxibustion.

\section{Treatment group intervention}

Participants in the treatment group will receive the same acupuncture treatment as the control group and will also take Palmijihwang-hwan. At visit 1, 4, and 8, they will receive 2-week doses of Palmijihwang-hwan (14 days + an extra 7 days) in an individual package from the practitioners. The Palmijihwang-hwan that will be used in this study is a brown, bitter herbal extract granule produced and packed by Kracie Pharma Korea Co., Ltd. (Seoul, Korea) in accordance with the Korean Good Manufacturing Practice guidelines. Palmijihwang-hwan contains eight herbs (listed in Table 2), is waterextracted with starch and lactose, and is regulated by the Korean Food \& Drug Administration. At the production stage, each herb is tested for properties, morphology, and undergoes physicochemical and microorganism examination. Then Palmijihwang-hwan is produced by extraction, separation, concentration, drying, and granulation with compound herbs. Multiple quality tests are performed, including pattern analysis of the ingredients contained to ensure quality and stability, and we will test for residual pesticides to ensure product safety. Palmijihwang-hwan, packed for one dose, will be provided by an independent pharmacist in a separate room and participants will be instructed to take it with water 30 min after breakfast and after dinner, for 6 weeks.

\section{Concomitant treatment}

Other interventions including muscle relaxants, nonsteroidal anti-inflammatory drugs (NSAIDs) (oral medication, topical application, or patch), antidepressant agents, anticonvulsants, treatments with Korean medicine (acupuncture, herbal medicine, and cupping), physical therapy, injections, or surgery will not be permitted during the treatment period (but are permitted during the follow-up period). However, concomitant medications taken within 4 weeks before trial participation or that are considered to have no effect on the interpretation of the results of this study may be allowed dependent on the judgment of the investigator.

\section{Adverse events}

Adverse events will be checked at every visit. If adverse events occur, the practitioners will record them in detail in the CRF and decide upon trial continuation depending on the symptoms. Abnormal change in blood chemistry tests, vital signs, or change of medication/treatment will also be checked.

\section{Outcome measurement Primary outcome}

The primary outcome of this study will be change in the VAS score between baseline (visit 1) and completion of the intervention (visit 12) [30]. Using the 10-cm VAS (0, absence of pain; 10, the worst pain imaginable), the participants will be asked to report their degree of LBP.

\section{Secondary outcomes}

\section{The secondary outcomes are:}

1. Pain: change in the VAS score between baseline (visit 1 ) and week 2 (visit 4), week 4 (visit 8), week 6 (visit 12), and 4 weeks after completion of the intervention (visit 13). Additional clinical relevance will be compared as follows;

a. Based on previous studies [31,32], the proportion of the minimal clinically important difference (MCID) in CLBP is defined as a $2 \mathrm{~cm}$ 


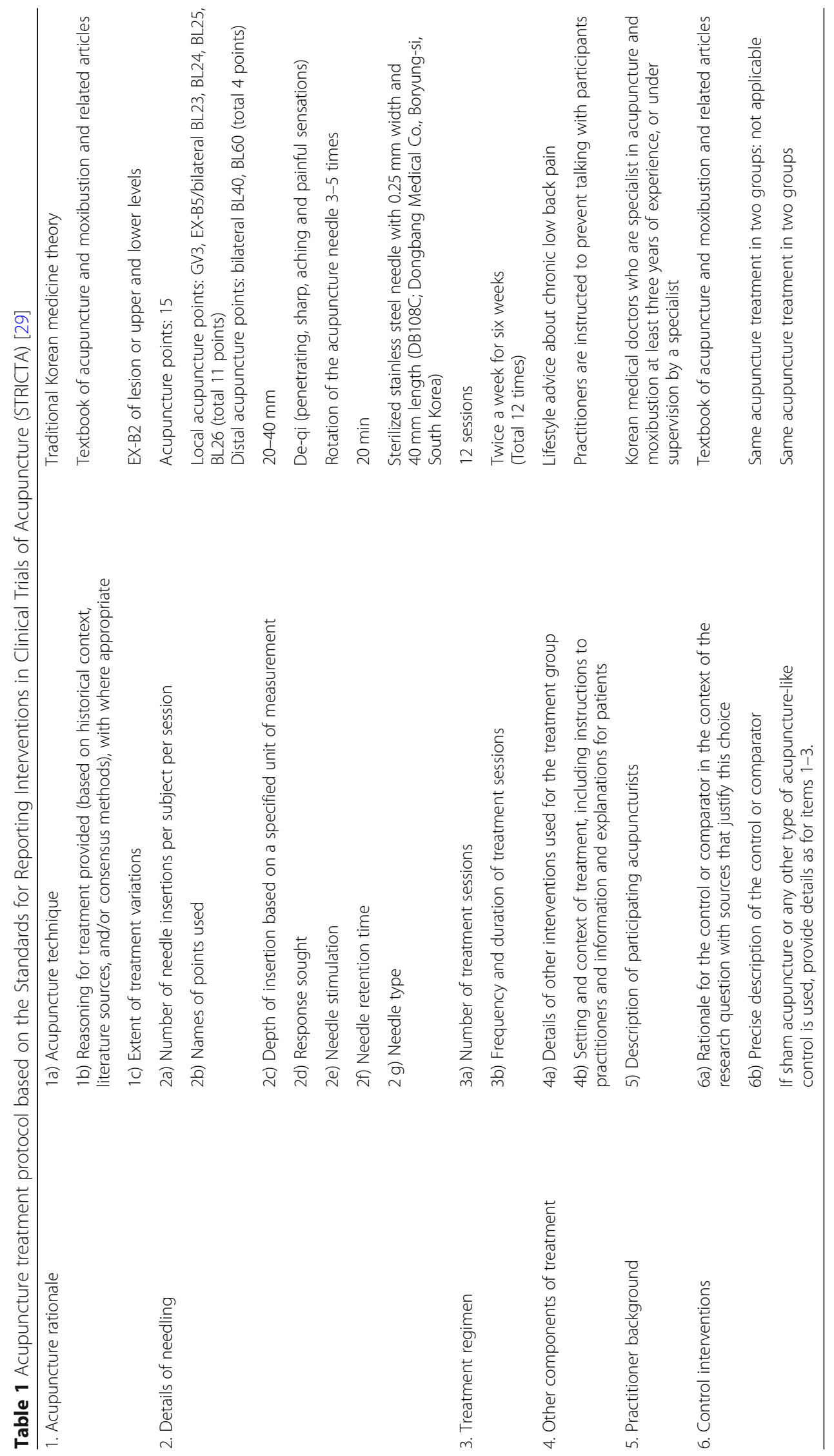


Table 2 Ingredients of Palmijihwang-hwan

\begin{tabular}{ll}
\hline Scientific name & Proportion \\
\hline Rehmannia glutinosa (steamed 9 times) & 5.0 \\
Cornus officinalis & 3.0 \\
Dioscorea batatas & 3.0 \\
Alisma orientale & 3.0 \\
Poria coccos & 3.0 \\
Paeonia sulffruticosa & 3.0 \\
Cinnamomum cassia & 1.0 \\
Aconitum carmichaelii (processed) & 1.0
\end{tabular}

decrease on the VAS. The proportion of MCID (more than $2 \mathrm{~cm}$ decrease on the VAS) between baseline and visit 4, 8, 12, and 13 will be recorded.

b. The proportion of participants who experience more than $30 \%$ decrease on the VAS between baseline and visit 4, 8, 12, and 13 .

c. The proportion of participants who experience more than $50 \%$ decrease on the VAS between the baseline and visit 4, 8, 12, and 13 .

2. Disability: to evaluate the physical disability due to low back pain, the Roland and Morris Disability Questionnaire (RMDQ) will be applied [33]. The RMDQ score is calculated by 24 items (in the range 0 (no disability) to 24 (maximum disability)). Change in score in the Korean version of the RMDQ will be compared between baseline and visit $4,8,12$, and 13 .

3. Quality of life: to evaluate quality of life, the EuroQol-5D (EQ-5D) questionnaire will be applied. The EQ-5D measures five dimensions (mobility, self-care, activities of daily life, pain, and anxiety/ depression) using a 1-3 scale, and a VAS to rate the current health state using a $0-100$ scale [34]; change in the EQ-5D scores between baseline and visit 4, 8, 12, and 13 will be compared.

4. Global assessment: to evaluate participants' impressions of change, the Patient Global Impression of Change (PGIC) will be applied at visit 12 and 13. The PGIC is measured on 7-point scale (from 1 (completely recovered) to 7 (vastly worsened)) with a score of 1-2 indicating improvement, 3-5 no change, and 6-7 deterioration [35].

5. Economic analysis: to evaluate the intervention-related cost, CEA and cost-utility analysis (CUA) will be conducted. Cost data will be collected separately as follows;

a. Medical cost: official medical costs will include benefit coverage by National health insurance and out-of-pocket payments such as for drugs and therapeutic equipment when visiting clinics or hospitals, for treating CLBP. Unofficial medical costs will include payments for over-the-counter drugs, dietary supplements, medical devices, orthotic devices, etc. which may be used without prescriptions by physicians.

b. Non-medical cost: transportation costs, care costs, time costs, and lost productivity costs.

6. Credibility test: to evaluate the credibility of Palmijihwang-hwan, a credibility/expectancy questionnaire will be applied at visit 1 . Using a 9point scale, a higher score indicates higher credibility and expectancy of Palmijihwang-hwan [36]

\section{Safety assessment}

Physical examinations will be performed at the screening visit and at visit 1 and 13. Blood chemistry tests including pregnancy tests will be conducted at the screening visit and visit 12 . The blood chemistry test will assess the red blood cell (RBC) count, white blood cell (WBC) count, hemoglobin $(\mathrm{Hb})$, hematocrit (Hct), platelet count, erythrocyte sedimentation rate (ESR), aspartate aminotransferase (AST), alanine aminotransferase (ALT), blood urea nitrogen (BUN), creatinine, electrolytes $(\mathrm{Na}, \mathrm{K}, \mathrm{Cl})$, and $\mathrm{C}$-reactive protein $(\mathrm{CRP})$. Vital signs, adverse events, and change in medication/treatment will be checked at every visit.

\section{Withdrawal criteria}

\section{The withdrawal criteria are:}

1. Violation of the inclusion criteria or applicable to exclusion criteria

2. Serious adverse events that make it difficult to maintain the trial

3. Refusal to continue, withdrawal of consent by the participants and/or legal representative

4. Compliance with Palmijihwang-hwan or acupuncture treatment less than $80 \%$ (participants should take more than 23 of the total 28 doses of Palmijihwang-hwan given at the time of the visit and receive more than 10 of the total 12 acupuncture treatments)

5. Violation of the clinical trial protocol by the investigator or the participants

6. Loss to follow up

7. Use of medications or treatments that affect the results of the trials without the permission from the practitioner

8. Inappropriate process as judged by the investigator 


\section{Data management and quality control}

To guarantee the consistency of the clinical trial among the institutions, all staff participating in this trial will receive training before study initiation. This training will include education about the procedures, acupuncture treatment, and outcome measure assessments.

A clinical research coordinator, trained in good clinical practice, will collect and record the study data in the CRF. To ensure confidence in the data, study-related documents including consent forms, the CRF, questionnaires, medical records, and other records will be stored in a locked space or on a password-protected computer in each hospital for 3 years after study completion.

If the protocol is revised, this will be handled by Kyung Hee University Korean Medicine Hospital as a central coordinating facility. Any protocol amendments will be reviewed and approved by the ethics committee before application in the study and published via the Clinical Research Information System (CRIS).

No formal data monitoring committee will be convened for this study. However, quality will be maintained in the study by an independent clinical research associate (CRA) who will regularly audit and monitor the study at each hospital. The CRA will monitor the study on site to protect participants' rights, assess compliance with the proto$\mathrm{col}$, and ensure the ongoing implementation of appropriate data entry and quality control procedures.

\section{Statistical analysis}

Continuous variables at baseline will be expressed as means plus/minus standard deviation and statistically tested using the two-sample $t$ test or the Wilcoxon rank sum test. Categorical variables at baseline will be expressed as frequencies and statistically tested using the chi-squared test or Fisher's exact test. If there is a significant difference between groups, the baseline variable will be considered as a covariate for efficacy analysis.

The full analysis set (FAS) will be analyzed primarily, including participants who take at least one dose of Palmijihwang-hwan and complete at least one pain assessment. Also, the per-protocol set (PPS) will be subordinately analyzed, including participants who take more than $80 \%$ of the total Palmijihwang-hwan dose and complete the trial. In the FAS analysis, the missing data will be imputed using the method of last observation carried forward. The primary outcome (change in the VAS score between baseline and endpoint) will be tested by analysis of covariance (ANCOVA) or rank ANCOVA (according to normality or homoscedasticity) with factors of group and study site and covariates of baseline values. Continuous variables for the secondary outcomes will also be tested, respectively, by ANCOVA or rank ANCOVA. Categorical variables for the secondary outcomes will be tested by chi-squared test or Fisher's exact test according to the number of the cells with a value of 5 or less in the contingency table. Every variable predefined in the outcome sections will be compared at each time point, respectively.

The safety set will include participants who receive at least one dose of Palmijihwang-hwan. In safety analysis, the frequency and ratio of adverse events will be applied and compared between the treatment group and the control group using the chi-squared test or Fisher's exact test. Any abnormal change in blood chemistry tests from visit 1 to 12 will be expressed as frequency and percentage and compared between the treatment group and the control group using McNemar's chi-squared test, and any change in vital signs between visit 1 and 12 and between visit 1 and 13 will be expressed as change scores and compared between the treatment group and the control group using the two-sample $t$ test or the Wilcoxon rank-sum test. Statistical tests will be carried out at 5\% significance level (two-sided), using $\mathrm{R}$ software (Version 3.5 .0 or later; $\mathrm{R}$ Foundation for Statistical Computing, Vienna, Austria).

For CEA, combination treatment with Palmijihwanghwan with acupuncture will be compared with acupuncture alone from a healthcare system perspective. The treatment cycle will be assumed to be 10 weeks. The treatment period (i.e. analysis period) can be the average life span if we build a model for the data analysis. If not, it is impossible to extrapolate the observations to the patient's life span. Therefore, this part is omitted as of now. For health-related outcomes, the RMDQ will be used for CEA and the EQ-5D for CUA. The absolute cost-effectiveness ratio will be estimated for the combination treatment of Palmijihwang-hwan with acupuncture and for acupuncture monotherapy. The cost-effectiveness of the combination therapy will be evaluated based on the incremental cost-effectiveness ratio. All economic analysis will be performed using TreeAgePro R 1.1 (Williamstown, MA, USA, 2018).

\section{Ethics approval and registration}

This study was approved by the Institutional Review Board of four hospitals (Kyung Hee University Korean Medicine Hospital; KOMCIRB-180413-HR-012, Kyung Hee University Korean Medicine Hospital at Gangdong; KHNMCOH 2018-04-001; Oriental Medicine Hospital of Daegu Haany University; DHUMC-D-18010-PRO-02, and Dongguk University Bundang Oriental Hospital; DUBOH 2018-0006), and registered in the Clinical Research Information Service (CRIS, KCT0002998) on 12 July 2018.

\section{Discussion}

Despite the development of medicine, the prevalence of CLBP and related disabilities have increased. Conventional treatments such as medications have been reported to have both benefits and harms [37], and several 
CAM therapies have been used widely and have proven efficacy and safety [38].

In herbal medicine, a CAM therapy, several randomized controlled trials have been conducted and Gagnier et al [39] have reviewed the effects of several herbal medicines on CLBP. However, these studies investigated the use of single herbal medicines such as Capsicum frutescens (cayenne) and Harpagophytum procumbens (devil's claw), and there has been no study of the complex herbal medicines that are mainly used in Korean medicine.

Palmijihwang-hwan was used in this study; this is a representative herbal formula for supplementing kidney deficiency in Korean medicine. Palmijihwang-hwan has been suggested to regulate immunity, peroxidation, and the aging process [40], and to have an effect on diverse diseases including diabetes mellitus [41], dementia [42], and chemotherapy-induced peripheral neuropathy [43].

In LBP, we suggested that Palmijihwang-hwan could help patients who have fatigue, cold sensation, lumbago, reduced muscle strength [44], constant pain, or discomfort behavior [45], which might be helpful in CLBP. In addition, kidney deficiency is one of the most commonly diagnosed causes of CLBP [46, 47]. In the absence of a study on the use of herbal medicine in CLBP, this study will be the opportunity to provide information on the usefulness of Palmijihwang-hwan. Pharmacologically, it may provide the clinical information about the toxicity of A. carmichaelii by safety assessment. The toxicity of A. carmichaelii was known that lowest oral dose was 0.2 $\mathrm{g} / \mathrm{kg}$ and processed aconitum carmichaelii was $60 \mathrm{~g} / \mathrm{kg}$ [48] while processed A. carmichaelii was $60 \mathrm{~g} / \mathrm{kg}$ [49].

Furthermore, the features of this study such as the multicenter design and various outcome measures, including clinical relevance, quality of life, disability level, global assessment, and economic analysis, will improve the quality of the trial data and prevent bias. However, this study has several limitations. First, participants in the control group cannot be blinded because of the absence of a placebo, which could influence the study results. Second, we quoted Lee's study for sample calculation but there is a difference in treatment duration. Participants in Lee's study [27] took Ojeok-san for 4 weeks, but this study needs participants to take Palmijihwang-hwan for 6 weeks. Third, there is no rescue therapy including drugs in the case of severe LBP, which could inhibit participants' compliance. However, the results of this study will provide clinical evidence about the efficacy, safety, and CEA of Palmijihwang-hwan for treatment of CLBP, and this evidence will be useful for patients, physicians, researchers, and stakeholders.

\section{Trial status}

The final protocol version is 2.1 and is dated 23 July 2018. Recruitment began on 17 August 2018 and is ongoing.

\section{Supplementary information}

Supplementary information accompanies this paper at https://doi.org/10. 1186/s13063-019-3776-7.

Additional file 1. SPIRIT 2013 Checklist: Recommended items to address in a clinical trial protocol and related documents.

\begin{abstract}
Abbreviations
ANCOVA: Analysis of covariance; CAM: Complementary and alternative medicine; CEA: Cost-effectiveness analysis; CLBP: Chronic low back pain; CRA: Clinical research associate; CRF: Case report form; CUA: Cost-utility analysis; EQ-5D: EuroQol-5 dimension; FAS: Full analysis set; LBP: Low back pain; MCID: Minimal clinically important difference; NSAID: Non-steroidal antiinflammatory drug; PGIC: Patient global impression of change; PPS: Perprotocol set; RMDQ: Roland and Morris disability questionnaire; STRICTA: Standards for reporting interventions in clinical trials of acupuncture; TNF: Tumor necrosis factor; VAS: Visual analog scale
\end{abstract}

\section{Authors' contributions}

$\mathrm{H}-J \mathrm{~L}, \mathrm{~B}-\mathrm{KS}$, and Y-CP contributed to the conceptualization of the study. DWN contributed to funding for the study. E-JK contributed to the study administration. SS investigated the design, methodology, and resources of the study. T-HK contributed to the study's statistical design. W-SS, S-RJ, and Y-JH prepared the study protocol and manuscript. W-SS reviewed and edited the study protocol and manuscript. D-WN supervised this study. All authors read and approved the final manuscript.

\section{Funding}

This study was supported by the Traditional Korean Medicine R\&D program funded by the Ministry of Health \& Welfare through the Korea Health Industry Development Institute (KHIDI): HB16C0040. The Sponsor has no role in the study design and will have no role in data collection, analysis, or interpretation; writing of the report; or the decision to submit the resulting report for publication.

\section{Availability of data and materials}

Data and material from this trial are available upon reasonable request and approval by the corresponding author.

\section{Ethics approval and consent to participate}

The protocol was written in accordance with the Declaration of Helsinki and Korean Good Clinical Practice. This study was approved by the Institutional Review Board of four hospitals and the final protocol version is 2.1 dated 23 July 2018. The protocol was registered in the Clinical Research Information Service (CRIS, KCT0002998) on 12 July 2018.

All participants will be informed about the trial, including design, potential benefits, and adverse events. Participants will be given enough time to decide on their participation and swill ign the informed consent form voluntarily.

\section{Consent for publication}

All authors reviewed the final manuscript, and consent for publication. There are no personal or private data contained in this manuscript.

\section{Competing interests}

The authors declare that they have no competing interests.

\section{Author details}

${ }^{1}$ Department of Acupuncture \& Moxibustion, Dongguk University Bundang Oriental Hospital, Bundang-gu, Seongnam-si, Gyeonggi-do 13601, South Korea. ${ }^{2}$ Department of Clinical Korean Medicine, Graduate School, Kyung Hee University, Seoul, South Korea. ${ }^{3}$ Clinical Trial Center, Korean Medicine Hospital, Department of Korean Medicine, Kyung Hee University, Seoul, South Korea. ${ }^{4}$ Department of Acupuncture \& Moxibustion, College of Korean Medicine, Daegu Haany University, Gyeongsan-si, Gyeongsangbuk-do, South Korea. ${ }^{5}$ Department of Acupuncture \& Moxibustion, College of Korean Medicine, Kyung Hee University, 26 Kyungheedae-ro, Dongdaemun-gu, Seoul 02447, South Korea. 
Received: 7 September 2018 Accepted: 9 October 2019

2

\section{References}

1. Balagué F, Mannion AF, Pellisé F, Cedraschi C. Non-specific low back pain. Lancet. 2012;379(9814):482-91.

2. Koes BW, van Tulder M, Lin CW, Macedo LG, McAuley J, Maher C. An updated overview of clinical guidelines for the management of non-specific low back pain in primary care. Eur Spine J. 2010;19(12):2075-94.

3. Croft PR, Macfarlane GJ, Papageorgiou AC, Thomas E, Silman AJ. Outcome of low back pain in general practice: a prospective study. BMJ. 1998; 316(7141):1356-9.

4. Andersson GB. Epidemiological features of chronic low-back pain. Lancet. 1999;354(9178):581-5.

5. Gaul C, Schmidt T, Czaja E, Eismann R, Zierz S. Attitudes towards complementary and alternative medicine in chronic pain syndromes: a questionnaire-based comparison between primary headache and low back pain. BMC Complement Altern Med. 2011;11:89.

6. Chou R, Huffman LH, American Pain Society; American College of Physicians. Nonpharmacologic therapies for acute and chronic low back pain: a review of the evidence for an American Pain Society/American College of Physicians clinical practice guideline. Ann Intern Med. 2007; 147(7):492-504.

7. Qaseem A, Wilt TJ, McLean RM, Forciea MA, Clinical Guidelines Committee of the American College of Physicians. Noninvasive treatments for acute, subacute, and chronic low back pain: a clinical practice guideline from the American College of Physicians. Ann Intern Med. 2017;166(7):514-30.

8. Toroski M, Nikfar S, Mojahedian MM, Ayati MH. Comparison of the costutility analysis of electroacupuncture and nonsteroidal antiinflammatory drugs in the treatment of chronic low back pain. J Acupunct Meridian Stud. 2018;11(2):62-6.

9. Seo BK, Han K, Kwon O, Jo DJ, Lee JH. Efficacy of bee venom acupuncture for chronic low back pain: a randomized, double-blinded, sham-controlled trial. Toxins (Basel). 2017;9(11):361.

10. Brinkhaus B, Witt CM, Jena S, Linde K, Streng A, Wagenpfeil S, et al. Acupuncture in patients with chronic low back pain: a randomized controlled trial. Arch Intern Med. 2006;166(4):450-7.

11. Witt CM, Jena S, Selim D, Brinkhaus B, Reinhold T, Wruck K, et al. Pragmatic randomized trial evaluating the clinical and economic effectiveness of acupuncture for chronic low back pain. Am J Epidemiol. 2006;164(5):487-96.

12. Cho YJ, Song YK, Cha YY, Shin BC, Shin IH, Park HJ, et al. Acupuncture for chronic low back pain: a multicenter, randomized, patient-assessor blind, sham-controlled clinical trial. Spine (Phila Pa 1976). 2013;38(7):549-57.

13. We JS, Won SH, Hwang JS, Sun SH, Ahn YM, Sohn SH, et al. The comparison of effectiveness between acupuncture and its cotreatment with direct moxibustion to low back pain patient. J Acupunct Res. 2004;21(8):221-31.

14. Yoo SM, Bae KY, Lee YH, Jung JJ, Lim SY. Research on the effect and satisfaction between independence and parallelism treatment of acupuncture and physical of lumbago patients. J Acupunct Res. 2005;22(6):75-84.

15. Jeong SY, Park ZW, Shin JM, Kim JY, Youn IY. The comparative study of effectiveness between acupuncture and its cotreatment with Calculus Bovis, Fel Ursi, Moschus pharmacopuncture on the treatment of acute low back pain. J Acupunct Res. 2011;28(4):105-10.

16. Takeda T, Tsuiji K, Li B, Tadakawa M, Yaegashi N. Proliferative effect of Hachimijiogan, a Japanese herbal medicine, in C2C12 skeletal muscle cells. Clin Interv Aging. 2015;10:445-51.

17. Hijikata Y. Analgesic treatment with Kampo prescription. Expert Rev Neurother. 2006;6(5):795-802.

18. Park G, Lee SH, Han JY, Oh DS. Altered TNF-a response by Aconibal ${ }^{\oplus}$ and methotrexate in a lipopolysaccharide-induced setting of inflammatory conditions: Potential on a synergistic combination. J Ethnopharmacol. 2018; 213:191-7.

19. Liou SS, Liu IM, Lai MC, Cheng JT. Comparison of the antinociceptive action of crude Fuzei, the root of Aconitum, and its processed products. J Ethnopharmacol. 2005;99(3):379-83.

20. Wang Y, Zhang R, Xie J, Lu J, Yue Z. Analgesic activity of catalpol in rodent models of neuropathic pain, and its spinal mechanism. Cell Biochem Biophys. 2014;70(3):1565-71.

21. Huang J, Zhang Y, Dong L, Gao Q, Yin L, Quan H, et al. Ethnopharmacology, phytochemistry, and pharmacology of Cornus officinalis Sieb. et Zucc. J Ethnopharmacol. 2018;213:280-301.
22. Jin M, Suh SJ, Yang JH, Lu Y, Kim SJ, Kwon S, et al. Anti-inflammatory activity of bark of Dioscorea batatas DECNE through the inhibition of iNOS and COX-2 expressions in RAW264.7 cells via NF-KB and ERK1/2 inactivation. Food Chem Toxicol. 2010;48(11):3073-9.

23. Ma Q, Han L, Bi X, Wang X, Mu Y, Guan P, et al. Structures and biological activities of the triterpenoids and sesquiterpenoids from Alisma orientale. Phytochemistry. 2016;131:150-7.

24. Schinella GR, Tournier HA, Prieto JM. Mordujovich de Buschiazzo P, Ríos IL. Antioxidant activity of anti-inflammatory plant extracts. Life Sci. 2002;70(9):1023-33.

25. Chou TC. Anti-inflammatory and analgesic effects of paeonol in carrageenanevoked thermal hyperalgesia. Br J Pharmacol. 2003;139(6):1146-52.

26. Liao JC, Deng JS, Chiu CS, Hou WC, Huang SS, Shie PH, et al. AntiInflammatory activities of Cinnamomum cassia constituents in vitro and in vivo. Evid Based Complement Alternat Med. 2012;2012:429320.

27. Lee SH. Randomized double blinded clinical trial of ojeoksan products extracted through different methods for low back pain. Kyung Hee University Graduate School. Thesis for the Degree of Master of Korean Medicine;Seoul. 2010:1-51.

28. Faul F, Erdfelder E, Buchner A, Lang AG. Statistical power analyses using G*Power 3.1: tests for correlation and regression analyses. Behav Res Methods. 2009:41 (4):1149-60.

29. MacPherson H, Altman DG, Hammerschlag R, Youping L, Taixiang W, White $A$, et al. Revised standards for reporting interventions in clinical trials of acupuncture (STRICTA): extending the CONSORT statement. PLoS Med. 2010;7(6):e1000261.

30. Hawker GA, Mian S, Kendzerska T, French M. Measures of adult pain: visual analog scale for pain (VAS Pain), Numeric Rating Scale for Pain (NRS Pain), McGill Pain Questionnaire (MPQ), Short-Form McGill Pain Questionnaire (SF$\mathrm{MPQ})$, Chronic Pain Grade Scale (CPGS), Short Form-36 Bodily Pain Scale (SF-36 BPS), and Measure of Intermittent and Constant Osteoarthritis Pain (ICOAP). Arthritis Care Res (Hoboken). 2011;63(Suppl 11):S240-52.

31. Todd KH, Funk JP. The minimum clinically important difference in physicianassigned visual analog pain scores. Acad Emerg Med. 1996;3(2):142-6.

32. Yuan J, Purepong N, Hunter RF, Kerr DP, Park J, Bradbury I, et al. Different frequencies of acupuncture treatment for chronic low back pain: an assessor-blinded pilot randomised controlled trial. Complement Ther Med. 2009;17(3):131-40.

33. Roland M, Fairbank J. The Roland-Morris Disability Questionnaire and the Oswestry Disability Questionnaire. Spine (Phila Pa 1976). 2000;25(24):3115-24.

34. Soer R, Reneman MF, Speijer BL, Coppes MH, Vroomen PC. Clinimetric properties of the EuroQol-5D in patients with chronic low back pain. Spine J. 2012;12(11):1035-9.

35. Maughan EF, Lewis JS. Outcome measures in chronic low back pain. Eur Spine J. 2010;19(9):1484-94.

36. Devilly GJ, Borkovec TD. Psychometric properties of the credibility/ expectancy questionnaire. J Behav Ther Exp Psychiatry. 2000;31(2):73-86.

37. Chou R. Huffman LH; American Pain Society; American College of Physicians. Medications for acute and chronic low back pain: a review of the evidence for an American Pain Society/American College of Physicians clinical practice guideline. Ann Intern Med. 2007;147(7):505-14.

38. Furlan AD, Yazdi F, Tsertsvadze A, Gross A, Van Tulder M, Santaguida L, et al. A systematic review and meta-analysis of efficacy, cost-effectiveness, and safety of selected complementary and alternative medicine for neck and low-back pain. Evid Based Complement Alternat Med. 2012;2012:953139.

39. Gagnier JJ, Oltean H, van Tulder MW, Berman BM, Bombardier C, Robbins CB. Herbal medicine for low back pain: a Cochrane Review. Spine (Phila Pa 1976). 2016;41(2):116-33.

40. Jiang W, Li S, Wang C, Wang Y. Comparative study of effects of three kinds of herbal mixture decoctions on improving immune senescence and free radical metabolism. Chin Med J. 1997;110(10):750-4.

41. Hirotani Y, Ikeda K, Myotoku M. Effects of the herbal medicine Hachimi-jiogan (Ba-Wei-Di-Huang-Wan) on insulin secretion and glucose tolerance in type 2 diabetic Goto-Kakizaki rats. Drug Discov Ther. 2010;4(2):129-34.

42. Iwasaki K, Kobayashi S, Chimura Y, Taguchi M, Inoue K, Cho S, et al. A randomized, double-blind, placebo-controlled clinical trial of the Chinese herbal medicine "ba wei di huang wan" in the treatment of dementia. J Am Geriatr Soc. 2004;52(9):1518-21.

43. Kimata Y, Ogawa K, Okamoto H, Chino A, Namiki T. Efficacy of Japanese traditional (Kampo) medicine for treating chemotherapy-induced peripheral neuropathy: a retrospective case series study. World I Clin Cases. 2016;4(10):310-7. 
44. Zhongjin Z. Jin Gui Yao Lüe. 2C or 3C AD (Han Dynasty). In: Luo XM, editor. English Edition. Beijing: New World Press; 1987.

45. Heo J. Donguibogam. Namsandang Seoul; 1980.

46. Sherman KJ, Hogeboom CJ, Cherkin DC. How traditional Chinese medicine acupuncturists would diagnose and treat chronic low back pain: results of a survey of licensed acupuncturists in Washington State. Complement Ther Med. 2001;9(3):146-53.

47. Hogeboom CJ, Sherman KJ, Cherkin DC. Variation in diagnosis and treatment of chronic low back pain by traditional Chinese medicine acupuncturists. Complement Ther Med. 2001;9(3):154-66.

48. Yang M, Ji X, Zuo Z. Relationships between the toxicities of Radix Aconiti Lateralis Preparata (Fuzi) and the toxicokinetics of its main DiesterDiterpenoid alkaloids. Toxins (Basel). 2018;10(10):391.

49. Zhou G, Tang L, Zhou X, Wang T, Kou Z, Wang Z. A review on phytochemistry and pharmacological activities of the processed lateral root of Aconitum carmichaelii Debeaux. J Ethnopharmacol. 2015;160:173-93.

\section{Publisher's Note}

Springer Nature remains neutral with regard to jurisdictional claims in published maps and institutional affiliations.

Ready to submit your research? Choose BMC and benefit from:

- fast, convenient online submission

- thorough peer review by experienced researchers in your field

- rapid publication on acceptance

- support for research data, including large and complex data types

- gold Open Access which fosters wider collaboration and increased citations

- maximum visibility for your research: over $100 \mathrm{M}$ website views per year

At $\mathrm{BMC}$, research is always in progress.

Learn more biomedcentral.com/submissions 